\title{
1 EDITORIAL
}

\section{Diagnosing postpartum endometritis in cattle}

3 I.M. Sheldon

4 Correspondence: Professor I Martin Sheldon BVSc DBR DCHP PhD FRCVS

5 Swansea University Medical School, Swansea University, Singleton Park, Swansea, 6 SA2 8PP, United Kingdom.

$7 \quad$ Email: i.m.sheldon@swansea.ac.uk Telephone 01792602709

9 Postpartum endometritis is a common cause of infertility in dairy cattle (Fig 1). Most

10 veterinarians can confidently examine the female reproductive tract to discriminate 11 between a cow with endometritis and a normal cow. However, veterinarians are less confident when asked what defines a case of endometritis, how to diagnose the disease, when after calving to make the diagnosis, and how sure they are that their method of diagnosis is accurate? These challenges are highlighted in a study by Kusaka and co-authors, comparing three methods for diagnosing postpartum endometritis, which is summarized on page $X X X$ of this week's issue of the Veterinary Record. ${ }^{1}$ The key finding was that between two and six weeks after calving there was little agreement amongst the three methods used to diagnose endometritis, but there was good agreement amongst the methods seven weeks after calving, when 20 identifying the mainly normal cows.

21 Endometritis is an important disease for veterinarians to be able to diagnose because 22 the disease causes infertility in individual cows, and reduces herd fertility. ${ }^{2-4}$

23 Endometritis increases the average interval from calving to first insemination by nearly 24 a week compared with normal cows, delays conception by about four weeks, and 25 nearly doubles culling for failure to conceive. This reduced fertility, even after the 
successful treatment of endometritis, is not only a consequence of the inflammation in

27 the uterus and oviduct during the disease, but also caused by abnormal oestrous cycles and damaged oocytes. ${ }^{5}$

29 The normal postpartum period includes prompt involution of the uterus and 30 regeneration of damaged endometrium, resumption of ovarian cyclical activity and the 31 ovulation of competent oocytes, and control of the pathogenic bacteria that are found 32 ubiquitously in the uterus of postpartum cattle. ${ }^{6}$ These concurrent processes take about three to four weeks, after which a normal cow usually has uterine horns $<3 \mathrm{~cm}$ diameter and a cervix $<5 \mathrm{~cm}$ diameter, with no pus detectable in the reproductive tract, and regular oestrous cycles. However, in about 15 to $20 \%$ of dairy cows there is pus in the reproductive tract and/or an enlarged cervix, which are signs of clinical endometritis. ${ }^{2 ;} 37$

The definitions of postpartum uterine disease are usually based on those proposed in 2006. ${ }^{8}$ Briefly, clinical endometritis is defined by the presence of pus in the uterus three weeks or more after calving, usually with a purulent uterine discharge detectable in the vagina, and/or a cervix $>7.5 \mathrm{~cm}$ diameter. The severity of endometritis can be scored based on the abundance and appearance of pus in the vaginal mucus: score 430 is normal, clear or translucent mucus; score 1 endometritis is mucus containing flecks of pus, which is usually an white or off-white colour; score 2 endometritis is mucus containing < 50\% pus; and, score 3 endometritis is mucus containing $>50 \%$ pus. ${ }^{4 ;} 8 ; 9$ The prognostic value of scoring the severity of endometritis is that animals with higher scores have lower treatment success rates and, even after successful treatment, they have lower conception rates. Subclinical endometritis is diagnosed when the uterine discharge is normal but the proportion of neutrophils in endometrial 
cytobrush samples exceed specified thresholds, which depend on the time after calving, as outlined in the present study. ${ }^{1}$

52 The methods used to diagnose endometritis usually rely on detecting the presence of 53 pus in the reproductive tract by inspection of the contents of the vagina, using a 54 gloved-hand, Metricheck device or vaginoscope, or by using transrectal 55 ultrasonography of the uterus (Fig 2). ${ }^{8 ;} 10$ The study by Kusaka and co-authors 56 compared the diagnosis of endometritis using a Metricheck device, transrectal ultrasonography, and counting the number of neutrophils in endometrial cytobrush samples. Despite each of these methods detecting an aspect of pus in the reproductive tract, there is often disagreement between operators; ${ }^{11}$ and, as reported in the present study and by others, there is disagreement between diagnostic methods. ${ }^{1 ; 4 ; 9 ; 10 ; 12}$ One explanations for this disagreement is probably the subjective nature of the diagnosis and scoring of endometritis. Furthermore, it is often possible to detect neutrophils in cytobrush samples when there is insufficient pus in the uterus 64 for a positive diagnosis by inspecting the contents of the vagina or ultrasonography of 65 the uterus. Similarly, it is sometimes possible to detect pus resulting from vaginitis or 66 cervicitis independently of endometritis. ${ }^{13}$ Indeed, in the study by Kusaka and coauthors, $43 \%$ of cases of endometritis diagnosed by ultrasound three weeks after calving did not have pus in the vagina detectable using the Metricheck device. ${ }^{1}$ Another challenge that the paper in the Veterinary Record helps to address is when to diagnose endometritis. The timing of diagnosis for endometritis has to allow three to

71 four weeks after parturition for recovery of the uterus in normal cows, and yet provide 72 sufficient time for treatment of diseased cows before insemination, which usually starts 73 from seven weeks after parturition. There is also a practical consideration about 
74 integrating the timing of diagnosis into routine fertility visits for dairy farms. The reduction in the proportion of cows with endometritis slowed after three to four weeks after calving in the study by Kusaka, ${ }^{1}$ which supports the widely applied clinical practice of examining cows for endometritis about four weeks after parturition. Thus, when visiting farms that have fortnightly routine fertility visits, veterinarians should examine cows for endometritis between three and five weeks after parturition.

Additional conclusions that can be drawn from the study in the Veterinary Record, ${ }^{1}$ are to be clear about the definitions of endometritis, and to score the severity of disease - if you can measure it, you can manage it. Furthermore, multiple lines of evidence provide for a more robust diagnosis, and veterinarians should use more than one diagnostic method. 1; 8; 9 First, herd records should be used to identify cows at risk of endometritis, such as cows with a history of retained foetal membranes, dystocia or twins. Second, the reproductive tract should be examined for the presence of pus using a hand, Metricheck or vaginoscope. Finally, transrectal ultrasonography should be used to identify pus in the uterus and whether there is an enlarged cervix.

Veterinarians are always going to be faced with the tensions between taking time to make an accurate diagnosis of endometritis and the need for efficient routine fertility visits to dairy herds. What helps is having a strategy to monitor postpartum dairy cows (see Box: What you need to know). Accurately detecting the presence of pus in the reproductive tract is important in postpartum cows, whether that pus may have come from disease or damage to the uterus, cervix or vagina. The future may bring more accurate diagnostics; for example, a cow-side test for neutrophils in the uterus would be handy. However, for now, there remains room for improvement in the veterinary diagnosis of postpartum endometritis in cows. 
References

99 1. KUSAKA H, HASEGAWA R, NISHIMOTO N, et al. Comparison of diagnostic

100 methods for uterine health in dairy cattle on different days postpartum. Vet Rec

1012020

102 2. LEBLANC SJ, DUFFIELD TF, LESLIE KE, et al. Defining and diagnosing

103 postpartum clinical endometritis and its impact on reproductive performance in dairy

104 cows. J Dairy Sci 2002;85:2223-2236

105 3. SHELDON IM, CRONIN J, GOETZE L, et al. Defining postpartum uterine

106 disease and the mechanisms of infection and immunity in the female reproductive

107 tract in cattle. Biol Reprod 2009;81:1025-1032

108 4. PLETICHA S, DRILLICH MHEUWIESER W. Evaluation of the metricheck

109 device and the gloved hand for the diagnosis of clinical endometritis in dairy cows. $J$

110 Dairy Sci 2009;92:5429-5435

111 5. BROMFIELD JJ, SANTOS JE, BLOCK J, et al. Physiology and endocrinology

112 symposium: Uterine infection: Linking infection and innate immunity with infertility in

113 the high-producing dairy cow. J Anim Sci 2015;93:2021-2033

114 6. SHELDON IM, CRONIN JC, BROMFIELD JJ. Tolerance and innate immunity

115 shape the development of postpartum uterine disease and the impact of endometritis

116 in dairy cattle. Annual Review of Animal Biosciences 2019;7:361-384

117 7. GERNAND E, REHBEIN P, VON BORSTEL UU, et al. Incidences of and

118 genetic parameters for mastitis, claw disorders, and common health traits recorded

119 in dairy cattle contract herds. J Dairy Sci 2012;95:2144-2156

120 8. SHELDON IM, LEWIS GS, LEBLANC S, et al. Defining postpartum uterine

121 disease in cattle. Theriogenology 2006;65:1516-1530 
122 9. SAVC M, DUANE M, O'GRADY LE, et al. Uterine disease and its effect on

123 subsequent reproductive performance of dairy cattle: A comparison of two cow-side

124 diagnostic methods. Theriogenology 2016;86:1983-1988

125 10. DE BOER MW, LEBLANC SJ, DUBUC J, et al. Invited review: Systematic

126 review of diagnostic tests for reproductive-tract infection and inflammation in dairy

127 cows. J Dairy Sci 2014;97:1-17

128 11. LEUTERT C, VON KRUEGER X, PLONTZKE J, et al. Evaluation of

129 vaginoscopy for the diagnosis of clinical endometritis in dairy cows. J Dairy Sci

$130 \quad 2012 ; 95: 206-212$

131 12. BARLUND CS, CARRUTHERS TD, WALDNER CL, et al. A comparison of

132 diagnostic techniques for postpartum endometritis in dairy cattle. Theriogenology

$133 \quad 2008 ; 69: 714-723$

134 13. DUBUC J, DUFFIELD TF, LESLIE KE, et al. Randomized clinical trial of

135 antibiotic and prostaglandin treatments for uterine health and reproductive

136 performance in dairy cows. J Dairy Sci 2011;94:1325-1338 


\section{WHAT YOU NEED TO KNOW}

- Accurate diagnosis of postpartum endometritis is important to justify treatment with antibiotics or hormones, and because these cows are less fertility than normal cows.

- Be clear about your definitions of endometritis and score the severity of disease.

- Aim to examine cows for endometritis between three and five weeks post partum - long enough for normal cows to recover after calving but soon enough to treat diseased cows before the insemination period.

- Use more than one diagnostic method. Use herd records to identify risk factors for endometritis; examine the genital tract for pus using a hand, Metricheck or vaginoscope; and, use ultrasonography to identify pus in the uterus and/or a cervix $>7.5 \mathrm{~cm}$ diameter.

- Be realistic about the accuracy of endometritis diagnosis. Re-examine cows two weeks later when the diagnosis is unclear or when cows are treated for disease. 


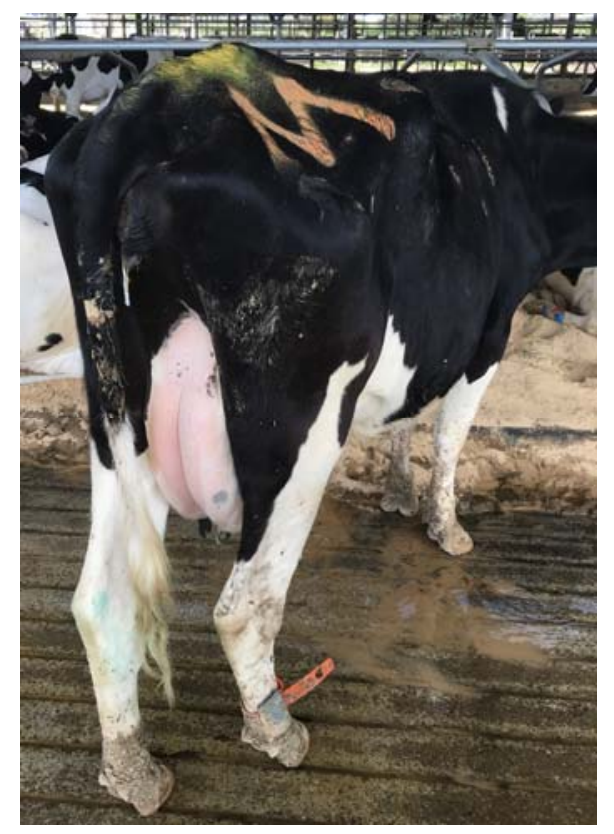

FIG1. Postpartum endometritis in a dairy cow.

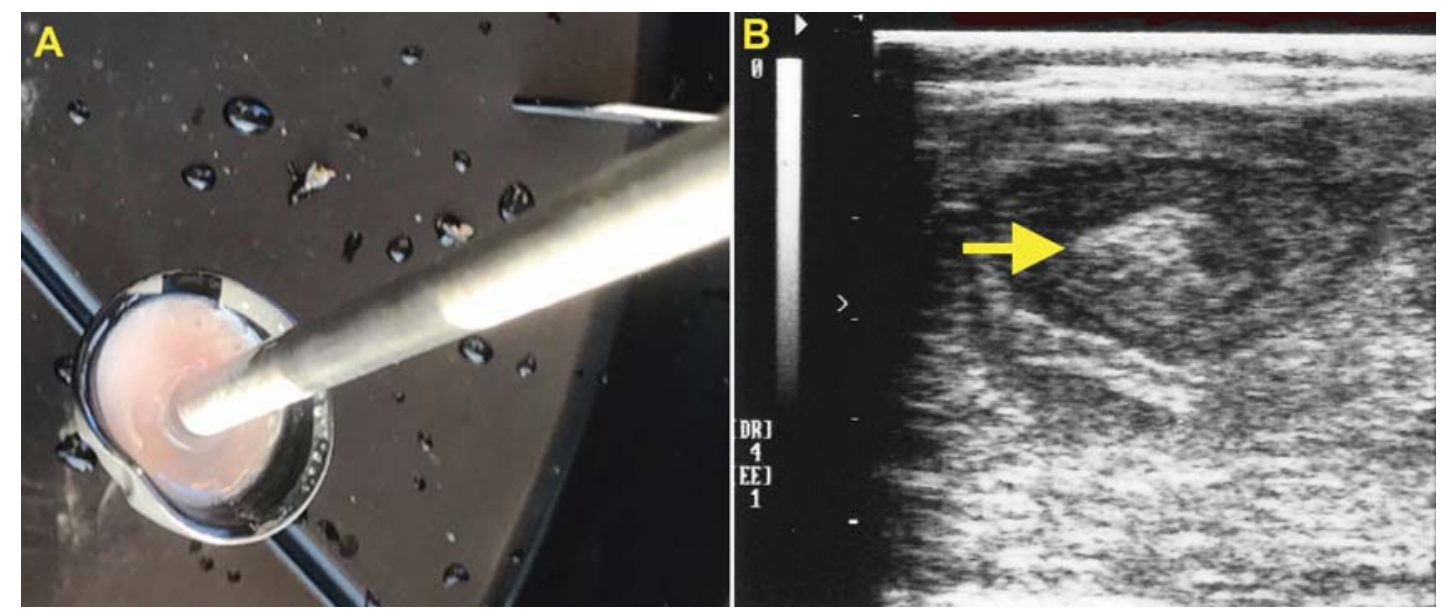

FIG 2. Diagnosis of endometritis. Common techniques for detecting the presence of pus in the reproductive tract about 4 weeks after calving include $(A)$ using the Metricheck device to collect mucus from the vagina, and (B) using transrectal ultrasonography of the uterine horns (arrow indicates pus in the uterine lumen). 